\title{
MASS TRANSFER ENHANCEMENT IN CHANNELS WITH A WAVY WALL
}

\author{
TATSUO NISHIMURA, YOSHIHIKO KAJIMOTO AND YUJI KAWAMURA \\ Department of Chemical Engineering, Hiroshima University, Higashi-Hiroshima 724
}

\begin{abstract}
Key Words: Fluid Mechanics, Friction Factor, Mass Transfer, Mass Transfer Enhancement, Wavy Channel, Electrochemical Method
\end{abstract}

A channel with wavy wall is one of several devices employed for enhancing the heat and mass transfer efficiency of processes having high Peclet numbers, such as the operation of compact heat exchangers, electodialysis and membrane blood oxygenators.

Recently, heat and mass transfer in wavy channels has been reported in several papers. There are two types of wavy channels in such problems: one is a channel with periodically converging-diverging cross section and straight flow axis, the other a channel with wavy flow axis and uniform cross section.

In the former case, Chow et al. ${ }^{1)}$ theoretically presented the local Nusselt number distributions in channels with a sinusoidal wavy wall at the entrance region in the laminar flow regime. Nishimura et al..$^{5)}$ analytically and experimentally obtained the relations between the Sherwood number and the Reynolds number for a similar geometrical channel in the laminar and turbulent flow regimes. They showed that this wavy channel is not an attractive enhancement device for laminar flow, but is attractive for turbulent flow as compared with a corresponding straight channel.

In the latter case, Sparrow et al. ${ }^{6)}$ experimentally obtained the Nusselt number in channels with a triangular wavy wall for turbulent flow. Consideration was given to the effects of varying the spacing between the wavy walls and of different fluid flow inlet conditions. Izumi et al. $^{2)}$ also calculated the local Nusselt number and wall shear stress distributions for various wavy channels in the laminar and turbulent flow regimes by a numerical calculation. However, it is not well known which of the former and latter channels is more effective in performance. This information is important to the design of enhancement devices.

This study is an extensive of the previous study. ${ }^{5}$ ) We considered the following three types of wavy channel as shown in Fig. 1, but the geometries of the

Received August 26, 1985 . Correspondence concerning this article should be addressed to T. Nishimura. Y. Kajimoto is now with Tokuyama Soda Co., Ltd., Tokuyama 745 . wavy wall are of the same: in the first, the crest of the lower wavy wall corresponds to the trough of the upper wavy wall of the channel $\left(\phi=180^{\circ}\right)$; in the second, one of the walls considered in the first has a phase-advanced $/ \operatorname{lag} \phi=90^{\circ}$ ); in the third, the crest of the lower wall corresponds to the crest of the upper wall $\left(\phi=0^{\circ}\right)$. Performance comparisons among these channels were carried out under equal pumping power constraint. The experiments covered the Reynolds number range from 100 to 10,000 .

\section{Experimental Apparatus and Procedure}

The experimental apparatus was the same as that used in the previous study. ${ }^{4}$ The test section consisted of a pair of sinusoidal wavy plates as shown in Fig. 1. The mean spacing between two plates was $13 \mathrm{~mm}$, yielding the cross-sectional aspect ratio of 15.38. Each wavy plate had an amplitude-to-length ratio of 0.25 and ten crests separated by a distance of $28 \mathrm{~mm}$.

Measurements of axial pressure drop were made using taps connected to $0.8 \mathrm{~mm}$ holes drilled through the lower wavy plate. Flow observations were performed by the hydrogen bubbles method. The detailed procedures were described in the previous study. ${ }^{4)}$ The fluid used was an aqueous solution of

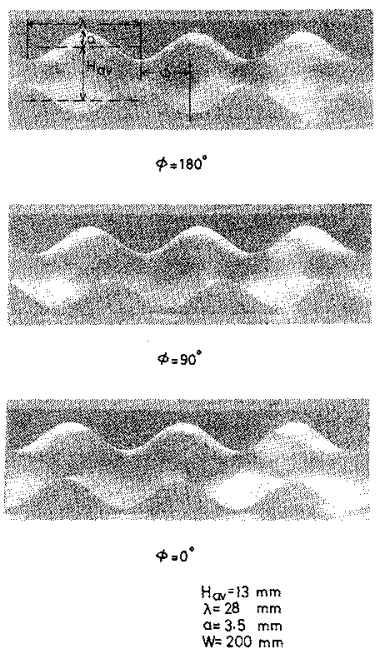

Fig. 1. Types of wavy channels. 
$40 \mathrm{wt} \%$ glycerol. The mass transfer coefficients were measured by an electrochemical method. Four electrodes $(L / \lambda=1,2,3$ and 4$)$ of the same types used in the previous study ${ }^{5}$ were located from the 6 th to 9 th wave in the lower plate, where the flow was fully developed. The electrolyte solution used contained $0.01 \mathrm{~N}$ potassium ferri-ferro cyanide and $1.0 \mathrm{~N}$ sodium hydroxide.

\section{Results and Discussion}

\subsection{Pressure drop}

Figure 2 shows the relationship between friction factor per unit wave length and Reynolds number in a fully developed flow region. The relationship for a straight channel is also included for reference. The friction factor of wavy channels is larger than that of the straight channel for a given Reynolds number, and this trend becomes more remarkable as the phase lag increases. For any wavy channel, the friction factor decreases with increasing Reynolds number up to about $R e=350$. Above this Reynolds number, the friction factor gradually increases and then tends to remain almost constant with increasing Reynolds number. These results suggest that the flow patterns for all the wavy channels remarkably change at about $R e=350$.

\subsection{Flow pattern}

Below about $R e=350$, steady vortices are formed near the crest of the upper wall and the trough of the lower wall for any wavy channel. As the Reynolds number increases further, the vortices change from steady to unsteady motion due to the vorticity concentration of the separated shear layer which lies on the boundary between the main stream and the vortex. Figure 3 shows photographs of the flow pattern in the wavy channels at $R e=350$ as an example. For any channel, the main stream undulated remarkably according to the unsteady vortex motion. Thus the friction factors mentioned above and the flow visualizations indicate that the transition point from laminar to turbulent flow occurs at about $R e=$ 350.

\subsection{Mass transfer}

The effect of mass transfer length is insignificant except in the laminar flow range $(R e<350)$ for any wavy channel, and is not shown here. Thus the Sherwood number for even $L / \lambda=1$ can be regarded as the cycle-averaged fully developed value in the turbulent flow regime. The reason for this is considered to be that the concentration boundary layer is destroyed by the unsteady vortex motion as observed by the flow visualizations and is periodically developed from the flow reattachment point in each section per unit wave length. The Sherwood numbers for different types of wavy channel are presented at $L / \lambda=3$ as an example in Fig. 4. The Sherwood numbers for a given

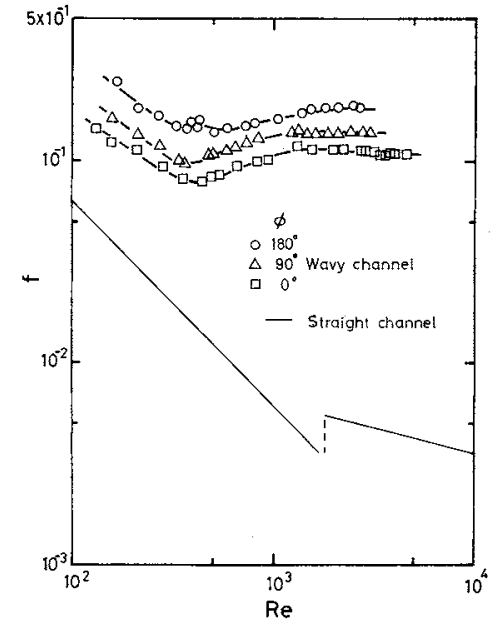

Fig. 2. Friction data for wavy channels.
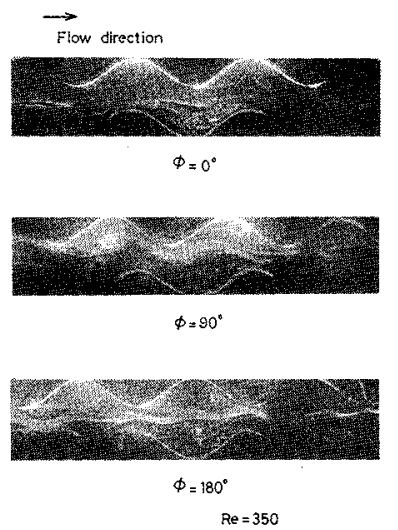

Fig. 3. Photographs of flow pattern for wavy channels at $R e=350$.

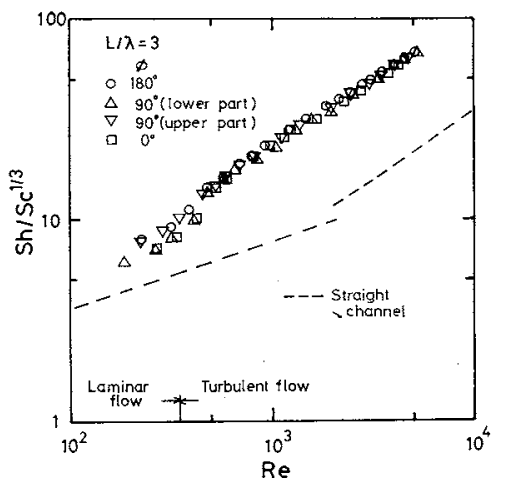

Fig. 4. Mass transfer data for wavy channels.

Reynolds number are almost equal for all the wavy channels in the turbulent flow regime, but are slightly different in the laminar flow regime. The effect of phase lag on mass transfer differs from that on the friction factor, which suggests that the Sherwood numbers for high Schmidt number $(S c=1170)$ are closely related to skin friction losses rather than inertial losses. The relationship for a straight channel is also included in this figure. The Sherwood number for wavy channels is larger than that for the straight 
channel.

\subsection{Performance evaluations}

The wavy and straight channels were compared under the condition of equal pumping power. The straight channel being compared has virtually identical dimensions (e.g. aspect ratio and spacing between the walls) to those of the wavy channels. Thus the mass transfer surface of the wavy channels increases to 1.14 times that in the straight channel case, which is not considered in performance evaluations. The friction factor and the Sherwood number for the straight channel were presented in the previous study ${ }^{5)}$ and are also shown in Figs. 2 and 4, respectively.

The constraint of equal pumping can be expressed as

$$
\left(f R e^{3}\right) \text { wavy channel }=\left(f R e^{3}\right) \text { straight channel }
$$

By using the friction factors shown in Fig. 2, the Reynolds numbers corresponding to equal pumping power for wavy and straight channels were calculated and with this Reynolds number relationship, the Sherwood numbers for the wavy and straight channels were determined from Fig. 4.* The ratio of Sherwood numbers, which corresponds to equal pumping power, is plotted in Fig. 5 as a function of $R e$, which is based the wavy channel. The Sherwood numbers for all the wavy channels exceed those for the straight channel. Thus, on a constant pumping power basis, these wavy channels can be regarded as enhancement devices. However, the effect of enhancement is limited within a certain range of Reynolds number. Further inspection of the figure shows that the greater enhancement occurs for channels with a smaller phase lag except in the lower Reynolds number range.

\section{Acknowledgment}

The authors acknowledge with thanks the assistance of Messers. A. Tarumoto and T. Yoshino. This work was supported in part by a Grant-in-Aid for Scientific Reserach (No. 5870092) from the Ministry of Education, Science and Culture of Japan.

\section{Nomenclature}

\begin{tabular}{|c|c|}
\hline$a$ & $=$ wave amplitude \\
\hline$f$ & $=$ friction factor $\left(=(1 / 4)\left(H_{\mathrm{av}} / \lambda\right)\left(2 \Delta P g / \rho u_{\mathrm{av}}^{2}\right)\right.$ \\
\hline$g$ & $=$ gravitational acceleration \\
\hline$H_{\mathrm{av}}$ & $=$ average spacing between wavy walls \\
\hline$k$ & $=$ mass transfer coefficient \\
\hline
\end{tabular}

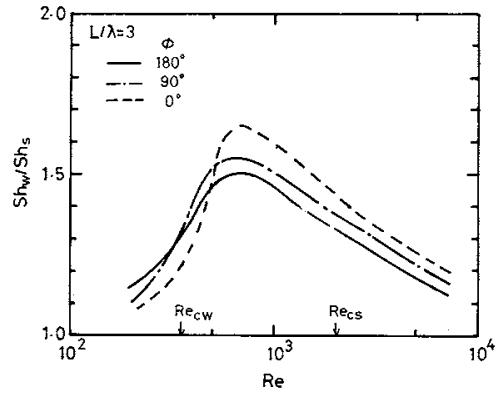

Fig. 5. Comparison of constant pumping power and Sherwood numbers for wavy and straight channels.

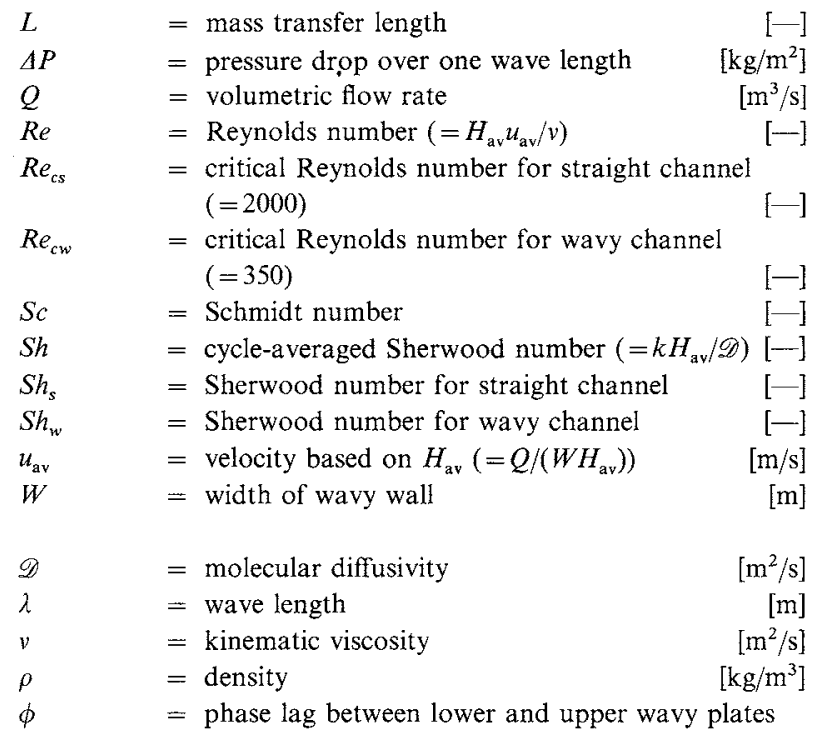

\section{Literature Cited}

1) Chow, J. C. F. and K. Soda: Trans. ASME J. Heat Transfer, 95, 352 (1973).

2) Izumi, R., H. Yamashita and K. Oyakawa: Trans. JSME, Ser. $B, 48,1994$ (1982)

3) Mendes, P. S. and E. M. Sparrow: Trans. ASME J. Heat Transfer, 106, 55 (1984).

4) Nishimura, T., Y. Ohori and Y. Kawamura: J. Chem. Eng. Japan, 17, 466 (1984).

5) Nishimura, T., Y. Ohori, Y, Kajimoto and Y. Kawamura: $J$. Chem. Eng. Japan, 18, 550 (1985).

6) Sparrow, E. M. and J. W. Comb: Int. J. Heat Mass Transfer, 26, 993 (1983)

(A part of this paper was presented at the 22nd National Heat Transfer Symposium of Japan, at Tokyo, May 1985.) 